\title{
Spatial distribution and determinants of iron supplementation among reproductive age women in Ethiopia: a spatial and multilevel analysis
}

Chilot Desta Agegnehu ( $\nabla$ chilotdesta@gmail.com )

University of Gondar College of Medicine and Health Sciences https://orcid.org/0000-0001-9407-4630

Getayeneh Antehunegn Tesema

University of Gondar College of Medicine and Health Sciences

Achamyeleh Birhanu Teshale

University of Gondar College of Medicine and Health Sciences

Adugnaw Zeleke Alem

University of Gondar College of Medicine and Health Sciences

Yigizie Yeshaw

University of Gondar College of Medicine and Health Sciences

Sewnet Adem Kebede

University of Gondar College of Medicine and Health Sciences

Alemneh Mekuriaw Liyew

University of Gondar College of Medicine and Health Sciences

\section{Research article}

Keywords: Iron supplementation use, spatial analysis, pregnant women, Ethiopia

Posted Date: June 11th, 2020

DOI: https://doi.org/10.21203/rs.3.rs-27876/v2

License: (a) (1) This work is licensed under a Creative Commons Attribution 4.0 International License. Read Full License 


\section{Abstract}

Background: Anemia remains a global public health problem. Despite WHO recommended all pregnant women to have iron supplementation, nearly $50 \%$ of maternal anemia in SSA was occurred due to lack of iron supplementation during pregnancy. Maternal anemia is associated with an increased risk of maternal death, obstetric complications, preterm birth, and low birth weight. There is limited evidence on the spatial pattern and determinants of iron supplementation using spatial and multilevel analysis methods. Therefore, this study aimed to investigate the spatial pattern, and determinants of iron supplementation in Ethiopia.

Methods: We used Ethiopian Demographic Health Survey in 2016 data explore the spatial distribution and associated factors of iron supplementation among women in Ethiopia. A weighted sample of 7589 women were included in the analysis. Bernoulli model was used to explore the purely spatial clusters of pregnant women using SaTScan version 9.6 and ArcGIS version10.3. Multilevel logistic regression analysis was fitted to identify significant individual and community level determinants of iron supplementation.

Results: Spatial analysis revealed that there was significant spatial variation of iron supplementation among reproductive age women in Ethiopia with Global Moran's index value of $0.3(p<0.001)$. The primary cluster was in Southwest Somali and Central part of the Oromia region ( $(L L R=66.69, P<0.001, R R=1.35)$. ANC visit $(A O R=3.66,95 \% \mathrm{Cl}: 3.21,417)$, community education $[A O R=1.31,95 \% \mathrm{Cl}, 1.07,1.59)$, media exposure $(A O R=1.33,95 \% \mathrm{Cl}: 1.15,1.53)$, distance to health facility (AOR=1.32, 95\% Cl: 1.16, 1.50), region and household wealth index were significantly associated with iron supplementation among women in Ethiopia.

Conclusion: The spatial distribution of iron supplementation among reproductive age women were significantly varied across the country with significant hotspot areas located in the Southwest Somali and Central Oromia regions. Besides, ANC visit, region, household wealth index, media exposure, distance to the health facility, and community education were significant determinants of iron supplementation among reproductive age women. Therefore, the finding of this study could help to design effective public health interventions targeting areas low iron supplementation and maternal health services should be delivered in all areas of our country. Keywords : Iron supplementation, spatial analysis, women, Ethiopia

\section{Background}

Globally, the prevalence of anemia among pregnant women is estimated to be $41.8 \%$ of which half this burden occurs due to iron deficiency or lack of iron tablet supplementation $(1,2)$. Iron deficiency anemia (IDA) is one of the foremost frequently observed preventable type of anemia among pregnant women's of developing countries $(2,3)$. The demand for iron during pregnancy increases due to either increased supply of iron to the fetus or its loss following delivery (4). For pregnant women the recommended iron supplementation is $20 \mathrm{mg}$ per day which is higher than the requirement for non-pregnant women (5). This is because the quantity of iron absorbed from the diet is not sufficient during pregnancy and infancy (6). Consistent with the advice of the World Health Organization (WHO), all pregnant women regardless of their hemoglobin level status should get iron supplementation (7). 
Anemia during pregnancy increases the risk of maternal death, obstetric complications, preterm birth, and low birth weight (8-10). Timely supplementation of the recommended dosage of prevents the after mentiond complications of maternal and child health $(11,12)$.

Factors associated with iron supplementation among pregnant women include: advanced maternal age(13, $14)$, parity (14), knowledge of about anemia and diagnosis of anemia $(14,15)$, and use of other food supplements (16). The coverage of iron supplementation among pregnant women is low in Ethiopia, below the recommendation of WHO (7).In addition it varies from region to region. Therefore, exploring the spatial distribution and associated factors of iron supplementation use among pregnant women is important for policymakers to take targeted intervention by identifying the most risky areas for the problem and factors associated with it. This targeted intervention would help to maximize the optimal utilization of scarce resources.

\section{Methods}

\section{Study setting}

The study was conducted in Ethiopia, the most Populus country in the horn of Africa. . The country covers 1.1 million square kilometers and has a great geographical diversity, which ranges from 4550 meters above water level right down to the Afar depression to 110 meters below the water level. There are nine regional states (list of regions in bracket) and two city administrations (Addis Ababa and Dire Dawa) subdivided into 68 zones, 817 districts, and 16,253 kebeles (lowest local administrative units of the country within the administrative structure of the country) (17).

\section{Data source, sampling procedure and study participants}

The study used data from the Ethiopian Demographic and Health Survey (EDHS) 2016, which was a community-based cross-sectional study conducted between January 18 to June 27, 2016. The EDHS2016 used a two-stage stratified cluster sampling technique to select study participants. In the first stage, a total of 645 EAs (202 in urban areas and 443 in rural areas) proportional to EA size were selected using 84915 enumeration areas from Ethiopian Population and Housing Census conducted in 2007 as a sampling frame. In the second stage, 28 households per each cluster were systematically selected (17). The women's (IR) data set was used for our study, and a total weighted sample of 7589 women who gave birth within 5 years preceding the survey.

\section{Variables of the study}

Iron supplementation use during pregnancy, coded as " 1 " if a pregnant woman was supplemented with iron and " 0 " if not supplemented with iron during pregnancy, was our outcome variable. After reviewing different literatures, for our study, both individual and community level variables were considered as independent/explanatory variables.

The individual level variables include - maternal age, religion, wealth index, occupational status, family size, and , mass media exposure, perception of distance from the health facility, ANC visit. 
The community level variables were residence, a region, and community level of women's education. Community level of women's education was created by aggregating the individual level factor women education since it was not directly found in the DHS data but known to a significant factor for the outcome variable. Since this variable was no normally distributed we used the median value to categorize it as low (communities with low proportions of women education) and high (communities with higher proportions of women education)

\section{Data management and analysis}

After downloading the info from the DHS program official database (www.measuredhsprogram.com), editing, recording, and analysis were done using STATA 14, ArcGIS 10.6, and SaTscan version 9.6. The data were weighted using sampling weight, primary sampling unit, and strata to restore the representativeness of the data and to get reliable estimate.

\section{Spatial analysis}

For the spatial analysis, ArcGIS version 10.6 and SaTScan version 9.6 statistical software were used for exploring the spatial distribution, global spatial autocorrelation, spatial interpolation, and for identifying significant hotspot areas of iron supplementation. The Global spatial autocorrelation was done to assess whether the spatial distribution of iron supplementation is random or not. The spatial autocorrelation (Global Moran's I) is the correlation coefficient for the relationship between a variable and its surrounding values. Moran's I is a spatial statistics used to measure spatial autocorrelation by taking the entire data set and produce a single output. Moran's I value ranges from-1 to 1 . A value close to 1 shows a strong positive spatial autocorrelation whereas a value close to -1 shows a strong negative spatial autocorrelation. If Moran's I close to 0 , it indicates that there is no spatial autocorrelation. A statistically significant Moran's I value $(p<0.05)$ can lead to rejection of the null hypothesis (iron supplementation is randomly distributed) and indicates the presence of spatial dependence.

Getis-OrdGi* statistics was used to identify significant hotspot and cold spot areas of iron supplementation. Getis-OrdGi* statistics were computed to measure how spatial autocorrelation that varies over the study location and Z-score is computed to determine the statistical significance of clustering, and the p-value computed for the significance. Statistical output with high Gl* indicates "hotspot" whereas low G/* means a "cold spot" .Kriging interpolation technique were employed to predict the prevalence of iron supplementation among reproductive age women based on the observed values. There are different types of interpolation techniques, for this study we choose ordinary Kriging interpolation techniques since it has small root mean square error and residuals.

Spatial scan statistical analysis was done using SaTscan version 9.6 software to identify significant primary and secondary clusters. Bernoulli model was fitted since the outcome variable was binary. A woman who didn't take iron supplementation were considered as cases whereas those who take iron supplementation were considered as control. The Bernoulli model require data for cases, controls, and geographic coordinates. The default maximum spatial cluster size of $<50 \%$ of the population was used, as an upper limit, since it allowed both small and large clusters to be detected and ignored clusters that contained more than 
the maximum limit. The scanning window with maximum likelihood was the most likely performing cluster, and the $p$-value was assigned to each cluster using Monte Carlo hypothesis testing by comparing the rank of the maximum likelihood from the real data with the maximum likelihood from the random datasets. The primary and secondary clusters were identified and assigned p-values and ranked based on their likelihood ratio test, based on 999 Monte Carlo replications (18).

\section{Multi-level analysis}

Due to the hierarchical nature of the DHS data, multilevel logistic regression analysis was done to identify the factors associated with iron supplementation use. The ICC (Intra-class correlation and MOR (median odds ratio) were computed to measure the variation between clusters/to assess the community level variability, and PCV (proportional change in variance) were calculated to assess how much iron supplementation use was explained by both individual and community level factors as follows $(19,20)$. Four models were constructed for the multi-level logistic regression analysis. The first model was an empty model without any explanatory variables, to calculate the extent of cluster variations on iron supplementation use among pregnant women. The second model was adjusted with individual-level variables, the third model was adjusted for community-level variables while the fourth was fitted with both individual and community level variables simultaneously. The fourth model was the best-fitted model since it had lower deviance and higher likelihood.

In the multivariable multilevel logistic regression analysis variables with a p-value $<0.05$ were considered as statistically significant. Adjusted Odds Ratio (AOR) with their corresponding 95\% confidence interval was determined to identify factors associated with iron supplementation use among pregnant women. After comparing all models a model with low deviance was considered as a fitted model. Multi-collinearity was also checked using the variance inflation factor (VIF). Accordingly, all variables had VIF $<5$ and tolerance greater than 0.1 indicating that there is no multi-collinearity.

\section{Ethical consideration}

Ethical approval was not that much necessary since we used publicly available data. But we got permission letter, to use the data set, from major demographic and health survey through the online request form http://www.dhsprogram.com.

\section{Results}

\section{Socio-demographic characteristics of study participants}

A total of 7589 respondents were included in the study. Above one fourth $2165(28.5 \%)$ of the respondents were within the age range of 25-29 years. About 1654 (21.8\%) respondents were from households with poorer wealth quantile category. Majority, $4406(58 \%)$ of the respondents perceived distance from the health facility as a big problem whereas only 2414 (32\%) had four or more ANC visit. loo media exposure, 2564(33.8\%) study participants had media exposure. Of all the respondents, $662(87.2 \%)$ were rural dwellers. Regarding region, about 3129 (41.2\%) were Oromia whereas only 33 (0.4\%) were from Dire Dawa. Moreover, about 4799 (63.2\%) of participants were from communities with lower community level of female education (Table 1). 


\section{Regional prevalence of iron supplementation among reproductive age women}

The prevalence of iron supplementation during pregnancy varies across the country. The highest and lowest prevalence of Iron supplementation during pregnancy was observed in Tigray $(77.2 \%)$ and Somali $(27.7 \%)$ regions respectively (Figure 1 ).

\section{Spatial analysis of Iron supplementation}

The spatial distribution of iron supplementation was non-random in Ethiopia with Global Moran's I value of $0.30(p<0.001)$ [Figure 3]. The high prevalence of iron supplementation was located in the Tigray, Northeast Amhara, Beneshangul Gumuz, Addis Ababa and Northeast SNNPR regions. Whereas areas with low prevalence of iron supplementations were identified in Somali, Afar, Southwest Oromia, West Gambella and Southwest part of Addis Ababa [Figure 2].

In the Getis OrdGi statistical analysis, significant hotspot areas (areas where iron supplementations was poor) were located in the Northeast Somali, South Afar, North West Gambela, West and east SNNPR, and Southwest Oromia regions (P-value<0.01). Whereas the significant cold spot areas (areas with high iron supplementation) were located in Tigray, North Amhara, East Addis Ababa, and North West Harari regions (Figure 3).

In the Kriging interpolation; the predicted high prevalence of iron supplementations were identified in the Northwest Gambela, east Somali, southwest Somali, North West Oromia, and Northeast Afar regions whereas, the predicted low prevalence of iron supplementation were identified in theTigray, Northwest Amhara, Northeast Addis Ababa, West Beneshangul Gumuz, Northeast Addis Ababa, and North SNNPR regions [Figure 4].

In the spatial scan statistics, a total of 271 significant clusters were identified, of these 89 were primary (most likely) clusters. The primary clusters were located in southwest Somali and central part of Oromia region centered at $5.330795 \mathrm{~N}, 41.837597 \mathrm{E}$ of geographic location with $441.87 \mathrm{~km}$ radius, a Relative Risk (RR) of 1.35 and Log-Likelihood ratio (LLR) of 66.68 , at $p<0.001$. . It showed that women within the spatial window had 1.35 times higher lokelihood of low iron supplementation than women outside the spatial window [Figure 5] [Table 2).

\section{Multilevel logistic regression analysis for assessing factors associated with iron supplementation among reproductive age women}

\section{Random effect analysis results}

The intraclass correlation within the null model indicated that $27 \%$ of the variability in iron supplementation was attributable to community level variability. In addition, the median odds ratio which was 2.9 in the null model indicates that there was variation of iron supplementation between clusters. If we randomly select women from two different clusters women at the cluster with higher iron supplementation had $2.9 \mathrm{~m}$ times higher odds of experiencing iron supplementation as compared with women at cluster with lower iron 
supplementation. Moreover, as shown by PCV in the final model, about $67 \%$ of the variability in iron supplementation was explained by both individual and community level factors (Table 3).

\section{The fixed effect analysis result}

Bi-variable multilevel logistic regression analysis was done to select variables for multivariable multilevel logistic analysis and Variable with a $\mathrm{p}$-value $<0.2$ were considered for multivariable analysis.

Of the four fitted models,the final multilevel logistic regression model (model 4) was the best-fitted model for this study because this model had high likelihood and low deviance.

In multivariable multilevel logistic analysis, individual-level factors like wealth index, ANC visit and media exposure were found to be significantly related to the odds of iron supplementation. Among the community level factors region, community education and perception of distance from the health facility were significantly related to iron supplementation.

Women who attended a minimum of four ANC visit had 3.66 times (AOR=3.66, 95\% $\mathrm{Cl}$ : 3.21, 417) higher odds iron supplementation use as compared to those they didn't attend the minimum requirement of ANC visit ( $<4$ ANC visit). The odds of iron supplementation was 1.33 times ( $\mathrm{AOR}=1.33,95 \% \mathrm{Cl}: 1.15,1.53)$ higher among those mothers who had media exposure as compared to their counterparts. Those mothers who were from poorer, middle and richer households had 1.33 (AOR=1.33, 95\%Cl: 1.15, 1.68), 1.39 (AOR=1.39, 95\% Cl: 1.10, 1.63), and $1.42(\mathrm{AOR}=1.13,95 \% \mathrm{Cl}$ : 1.131.76) times higher odds of iron supplementation as compared with women from poorer households. Regarding perception of distance from the health facility, women who perceived distance from the health facility had $1.32(\mathrm{AOR}=1.32,95 \% \mathrm{Cl}: 1.16,1.50)$ times higher odds of iron supplementation as compared to their counterparts.

With adjusting other covariates, women's in Tigray, Afar, Amhara, and Dire Dawa regions were 5.35 $(A O R=5.35,95 \% \mathrm{Cl}: 3.73,7.69), 1.61(\mathrm{AOR}=1.61,95 \% \mathrm{Cl}: 1.13,2.28), 2.07(\mathrm{AOR}=2.07,95 \% \mathrm{Cl}: 1.47,2.91)$ and $1.54(\mathrm{AOR}=1.54,95 \% \mathrm{Cl}: 1.03,2.33)$ times higher iron supplementation use than that of women's in Somali region, respectively

Moreover, women from communities with higher women education had 1.31 [AOR= 1.31, 95\% Cl, 1.07, 1.59) times higher odds of iron supplementation as compared to those women from lower women education (Table 3).

\section{Discussion}

The spatial analysis revealed that the spatial distribution of iron supplementation among reproductive age women was significantly varied across the country. The significant hotspot areas with low iron supplementation were located in Northeast Somali, South Afar, North West Gambela, West and east a part of SNNPR, and Southwest Oromia regional states were statistically significant hot spot areas for iron supplementation (low iron supplementation) and the SaTscan analysis identifies significant primary (Most likely clusters) clusters in southwest Somali and central part of Oromia region. The possible explanation might be due to the Lowest ANC utilization rate was reported in hot spot areas as compared to cold spot 
areas lowest ANC service utilization in the border areas of the country (17), and the. This might be attributed to the discrepancy within in the distribution of maternal health service, and inaccessibility of infrastructure within in the border areas of Somali, and Benishangul regions (21). Furthermore, These regions also are relatively rural residents and that they couldn't access health facilities and ladies might not have awareness about the iron supplementation program and its benefit during their pregnancymight not have good access to maternal health care services. In several studies also evidence showed that in Somali,Hareri, and Afar regions the prevalence of anemia among reproductive-age women was high $((22,23)$. The finding of multilevel multivariable logistic regression indicated that ANC visits, community women's education, region, media exposure, household wealth index, and distance to the clinic as associated factors of iron supplementation use among pregnant women in Ethiopia.

ANC visit were significant determinants of iron supplementation. Women who had 4 and above ANC visit during pregnancy had higher odds of iron supplementations as compared to women who didn't have ANC visit during pregnancy. This was consistent with study findings reported in study is supported by a study wiped out (13), Tanzania (24), Senegal (15), Pakistan (25), and India (14). The possible justification might be mothers who had adequate ANC visits (four and above) may have information about the importance of iron supplementation and should have a positive attitude towards maternal health services including iron use compared to their counterparts. In this study, community women's education is one of the important factors for iron supplementation among pregnant women. The chance of getting an iron tablet is 1.3 times higher among women with higher community education as compared to lower community education. The finding of this study is similar with the finding of studies in India (14), Pakistan (25), and Senegal (26). This could be due to education is a vital tool to enhance knowledge of pregnant women about the consequence of iron deficiency and show the ways to handle these deficiencies. This means educated pregnant women have an excellent ability to take maternal health services like iron supplementation during pregnancy (27-29).

Media exposure is another factor associated with iron supplementation among pregnant women. Pregnant women who were exposed to media were 1.3 times more likely to take iron tablets as compared to their counterparts. This study was supported by a study in Asia (30). This is due to the fact that different maternal health services including the importance of taking iron during pregnancy is frequently given for the community through mass media. Therefore, pregnant women who exposed to media would have a far better understanding of the advantages of taking iron tablets compared to those who do not have media exposure.

Region is also significantly associated with iron supplementation among pregnant women. Those pregnant women from Tigray, Afar, Amhara, and Dire Dawa regions were 5.35, 1.6, 2, and 1.5 times higher chance of taking iron tablet as compared to Somali region. This could be explained by the difference in the coverage of ANC utilization across these regions, lower ANC visit was observed $w$ in the Somali region compared to other regions (17). This is often the very fact that ANC visits are the major route to deliver iron supplementation for pregnant women in Ethiopia. That's why pregnant mothers in Tigray, Afar, Amhara, and Dire Dawa regions had better iron supplementation than the Somali region.

The household wealth index is another important factor of iron supplementation among pregnant women. Mothers who were in poorer, middle, and richer wealth quantile categories were nearly 1.4 times higher chance of taking iron supplementation as compared within the poorest wealth quantile. This study finding is 
consistent with a study finding reported in Senegal (26). The possible explanation could be due to the reason that richest women have good access to maternal health care service utilizations (31). In this study, distance to health facility is significantly associated with iron supplementation. The odds of iron supplementation among women where distance to health facility was not a big problem were higher as compared to women who had big problem to reach health facility. This might be due to the reason that women who can easily accessing health care could have higher likelihood of utilizing maternal health care services like ANC visit. The strengths of this study, first, the study was based on a nationally representative weighted data and can be generalized at national level. Second, the use of spatial analysis to explore the spatial distribution and significant hotspot areas of iron supplementations and use of multilevel analysis. The limitations of this study were, it shares the limitations of cross-sectional studies.

\section{Conclusion}

This study investigated that the spatial clustering of poor iron supplementation among pregnant women was found in northeast Somali, south Afar, northwest Gambela, western, and eastern part of SNNPR and southwest Oromia regions. Community education, household wealth index, ANC visit, region, media exposure, and distance to a health facility were significant predictors of iron supplementation among pregnant women. To enhance iron supplementation, targeted intervention should be taken on these identified high risky areas. Intervention programs that can increase the frequency of ANC visit, education level, and use of mass media need to be done.

\section{Declarations}

\section{Ethical approval}

Permission for data access was obtained from major demographic and health survey through the online request from http://www.dhsprogram.com.

\section{Consent for publication}

Not applicable

\section{Availability of Data and Materials}

All relevant data are included within the manuscript. It also possible to access the data set online from DHS program

\section{Competing interest}

All authors declare that they have no competing interest.

\section{Funding}

The authors received no specific funding for this work. 


\section{Author's Contribution}

$\mathrm{CDA}, \mathrm{ABT}$, and GAT were involved in the design and conception of the study; the analysis and interpretation of the findings, and write the manuscript. SAK, YY, AZA, and AML involved in the analysis, interpretation, and writes up of the manuscript. All the authors read and approved the final manuscript.

\section{Acknowledgments}

We would like to acknowledge MEASURE DHS program for permitting data access and central statistical agency for giving the shapefile for this study.

\section{Abbreviations}

AIC: Akaike information criteria

ANC: Antenatal Care

AOR: Adjusted Odds Ratio

BIC: Bayesian information criteria

COR: Crude Odds Ratio

EDHS: Ethiopian Demographic health survey

ICC: Intra-Class Correlation

MOR: Median Odds Ratio

PCV: Proportional Change in Varianc

\section{References}

1. Stevens GA, Finucane MM, De-Regil LM, Paciorek CJ, Flaxman SR, Branca F, et al. Global, regional, and national trends in haemoglobin concentration and prevalence of total and severe anaemia in children and pregnant and non-pregnant women for 1995-2011: a systematic analysis of populationrepresentative data. The Lancet Global Health. 2013;1(1):e16-e25.

2. WHO C. Worldwide prevalence of anaemia 1993-2005. WHO global database on anaemia. 2008.

3. Yekta Z, Ayatollahi H, Pourali R, Farzin A. Predicting factors in iron supplement intake among pregnant women in urban care setting. Journal of research in health sciences. 2008;8(1):39-45.

4. Bothwell TH. Iron requirements in pregnancy and strategies to meet them. The American journal of clinical nutrition. 2000;72(1):257S-64S.

5. Trumbo P, Yates AA, Schlicker S, Poos M. Dietary reference intakes: vitamin A, vitamin K, arsenic, boron, chromium, copper, iodine, iron, manganese, molybdenum, nickel, silicon, vanadium, and zinc. Journal of the Academy of Nutrition and Dietetics. 2001;101(3):294. 
6. Stoltzfus RJ, Dreyfuss ML. Guidelines for the use of iron supplements to prevent and treat iron deficiency anemia: Ilsi Press Washington, DC; 1998.

7. Organization WH. Daily iron and folic acid supplementation in pregnant women: guideline. Daily iron and folic acid supplementation in pregnant women: guideline2012.

8. Allen LH. Anemia and iron deficiency: effects on pregnancy outcome. The American journal of clinical nutrition. 2000;71(5):1280S-4S.

9. Milman N. Oral iron prophylaxis in pregnancy: not too little and not too much! Journal of pregnancy. $2012 ; 2012$.

10. Menon KC, Ferguson EL, Thomson CD, Gray AR, Zodpey S, Saraf A, et al. Effects of anemia at different stages of gestation on infant outcomes. Nutrition. 2016;32(1):61-5.

11. Peña-Rosas JP, De-Regil LM, Garcia-Casal MN, Dowswell T. Daily oral iron supplementation during pregnancy. Cochrane database of systematic reviews. 2015(7).

12. Haider BA, Olofin I, Wang M, Spiegelman D, Ezzati M, Fawzi WW. Anaemia, prenatal iron use, and risk of adverse pregnancy outcomes: systematic review and meta-analysis. Bmj. 2013;346:f3443.

13. Haile D, Tabar L, Lakew Y. Differences in spatial distributions of iron supplementation use among pregnant women and associated factors in Ethiopia: evidence from the 2011 national population based survey. BMC pregnancy and childbirth. 2017;17(1):33.

14. Roy MP, Mohan U, Singh SK, Singh VK, Srivastava AK. Socio-economic determinants of adherence to iron and folic acid tablets among rural ante-natal mothers in Lucknow, India. National Journal of Community Medicine. 2013;4(3):386-91.

15. Gebremedhin S, Samuel A, Mamo G, Moges T, Assefa T. Coverage, compliance and factors associated with utilization of iron supplementation during pregnancy in eight rural districts of Ethiopia: a crosssectional study. BMC public Health. 2014;14(1):607.

16. Demuth IR, Martin A, Weissenborn A. Iron supplementation during pregnancy-a cross-sectional study undertaken in four German states. BMC pregnancy and childbirth. 2018;18(1):491.

17. Central Statistical Agency (CSA) Ethiopia. Demographic and health survey 2016. Addis Ababa, Ethiopia and Calverton, Maryland, USA: CSA and ORCMacro; . 2016.

18. Kulldorff M. SaTScanTM user guide. Boston[Google Scholar]. 2006.

19. Rodriguez G, Elo I. Intra-class correlation in random-effects models for binary data. The Stata Journal. 2003;3(1):32-46.

20. Merlo J CB, Ohlsson H, Beckman A, Johnell K, Hjerpe P, et al. A brief conceptual tutorial of multilevel analysis in social epidemiology: using measures of clustering in multilevel logistic regression to investigate contextual phenomena. Journal of Epidemiology \& Community Health. 2006;60(4):290-7.

21. Adugna A. Health instituition and Services in Ethiopia. . 2014.

22. Kibret KT, Chojenta C, D’Arcy E, Loxton D. Spatial distribution and determinant factors of anaemia among women of reproductive age in Ethiopia: a multilevel and spatial analysis. BMJ open. 2019;9(4):e027276.

23. Ejigu BA, Wencheko E, Berhane K. Spatial pattern and determinants of anaemia in Ethiopia. PloS one. 2018;13(5). 
24. Ogundipe O, Hoyo C, Østbye T, Oneko O, Manongi R, Lie RT, et al. Factors associated with prenatal folic acid and iron supplementation among 21,889 pregnant women in Northern Tanzania: a cross-sectional hospital-based study. BMC public health. 2012;12(1):481.

25. Nisar YB, Dibley M, Mir A. Factors Associated with Non-use of Antenatal Iron and Folic Acid Supplements among Pakistani Women: A Cross Sectional Household Survey. European Journal of Nutrition \& Food Safety. 2015:703-4.

26. Niang K, Faye A, Tine JAD, Diongue FB, Ndiaye B, Ndiaye MB, et al. Determinants of iron consumption among pregnant women in southern Senegal. Open Journal of Obstetrics and Gynecology. 2016;7(1):4150.

27. Raut BK, Jha MK, Shrestha A, Sah A, Sapkota A, Byanju S, et al. Prevalence of iron deficiency anemia among pregnant women before iron supplementation in Kathmandu university Hospital/Dhulikhel Hospital. J Gynecol Obstet. 2014;2(4):54-8.

28. Agrawal S, Fledderjohann J, Vellakkal S, Stuckler D. Adequately diversified dietary intake and iron and folic acid supplementation during pregnancy is associated with reduced occurrence of symptoms suggestive of pre-eclampsia or eclampsia in Indian women. PloS one. 2015;10(3):e0119120.

29. Gebreamlak B, Dadi AF, Atnafu A. High adherence to iron/folic acid supplementation during pregnancy time among antenatal and postnatal care attendant mothers in Governmental Health Centers in Akaki Kality Sub City, Addis Ababa, Ethiopia: Hierarchical negative binomial poisson regression. PloS one. 2017;12(1):e0169415.

30. Warvadekar K, Reddy J, Sharma S, Dearden KA, Raut MK. Socio-demographic and economic determinants of adherence to iron intake among pregnant women in selected low and lower middle income countries in Asia: insights from a cross-country analyses of global demographic and health surveys. International Journal Of Community Medicine And Public Health. 2018;5(4):1552-69.

31. Ethiopia MoHo. Ethiopia's Fourth National Health Accounts, 2007/2008. Addis Ababa: Ministry of Health [Ethiopia]. 2010.

\section{Tables}

Table 1:- Percentage distribution of characteristics of respondents in 2016 Ethiopian Demographic and Health Surveys. 


\begin{tabular}{|c|c|c|}
\hline Characteristics & Weighted frequency & Percentage \\
\hline \multicolumn{3}{|l|}{ Age } \\
\hline $15-19$ & 339 & 4.5 \\
\hline $20-24$ & 1,465 & 19.3 \\
\hline $25-29$ & 2,165 & 28.5 \\
\hline $30-34$ & 1,661 & 22 \\
\hline $35-39$ & 1,206 & 16 \\
\hline $40-44$ & 546 & 7 \\
\hline $45-49$ & 207 & 2.7 \\
\hline \multicolumn{3}{|l|}{ Residence } \\
\hline Rural & 6620 & 87.2 \\
\hline Urban & 969 & 12.8 \\
\hline \multicolumn{3}{|l|}{ Religion } \\
\hline Orthodox & 2,882 & 38 \\
\hline Protestant & 1,651 & 21.8 \\
\hline Muslim & 2,824 & 37.2 \\
\hline Others* & 232 & 3 \\
\hline \multicolumn{3}{|l|}{ Marital status } \\
\hline Single & 144 & 2 \\
\hline Married & 7020 & 92.5 \\
\hline Widowed & 95 & 1.2 \\
\hline Divorced & 233 & 3 \\
\hline Separated & 97 & 1.3 \\
\hline \multicolumn{3}{|l|}{ Region } \\
\hline Somali & 269 & 3.5 \\
\hline Tigray & 537 & 7.1 \\
\hline Afar & 71 & 1 \\
\hline Amhara & 1,632 & 21.5 \\
\hline Oromia & 3,129 & 41.2 \\
\hline Benishangul-Gumuz & 81 & 1.1 \\
\hline SNNPR & 1,600 & 21.1 \\
\hline & e $13 / 26$ & \\
\hline
\end{tabular}


Gambelia

Harari

Addis Ababa

Dire Dawa

Wealth index

Poorest

Poorer

Middle

Richer

Richest
21

0.3

17.4

198.3

33.3

1,651

1,654

1,588

1,427

1,269

Community women's education

Lower community education

4799

63.2

Higher community education 2790

36.8

Occupational status

Not working

4,078

53.7

Working

3,511

46.3

Distance to health facility

A big problem

4,406

58

Not a big problem

3,183

42

Parity

$0-4$

5-9

$10+$

4,624

2,732

233

Family size

1-4

2331

4,875

383

$10+$

ANC visit

0-3 times

5,175

4+times

2,414
68

61

36

3

31

64

5

32

Media exposure 
Not have media exposure $5025 \quad 66.2$

Have media exposure $\quad 2,564 \quad 33.8$

*key: others- traditional, catholic

Table 2: Sat Scan analysis of iron supplementation among pregnant women in Ethiopia, 2016 


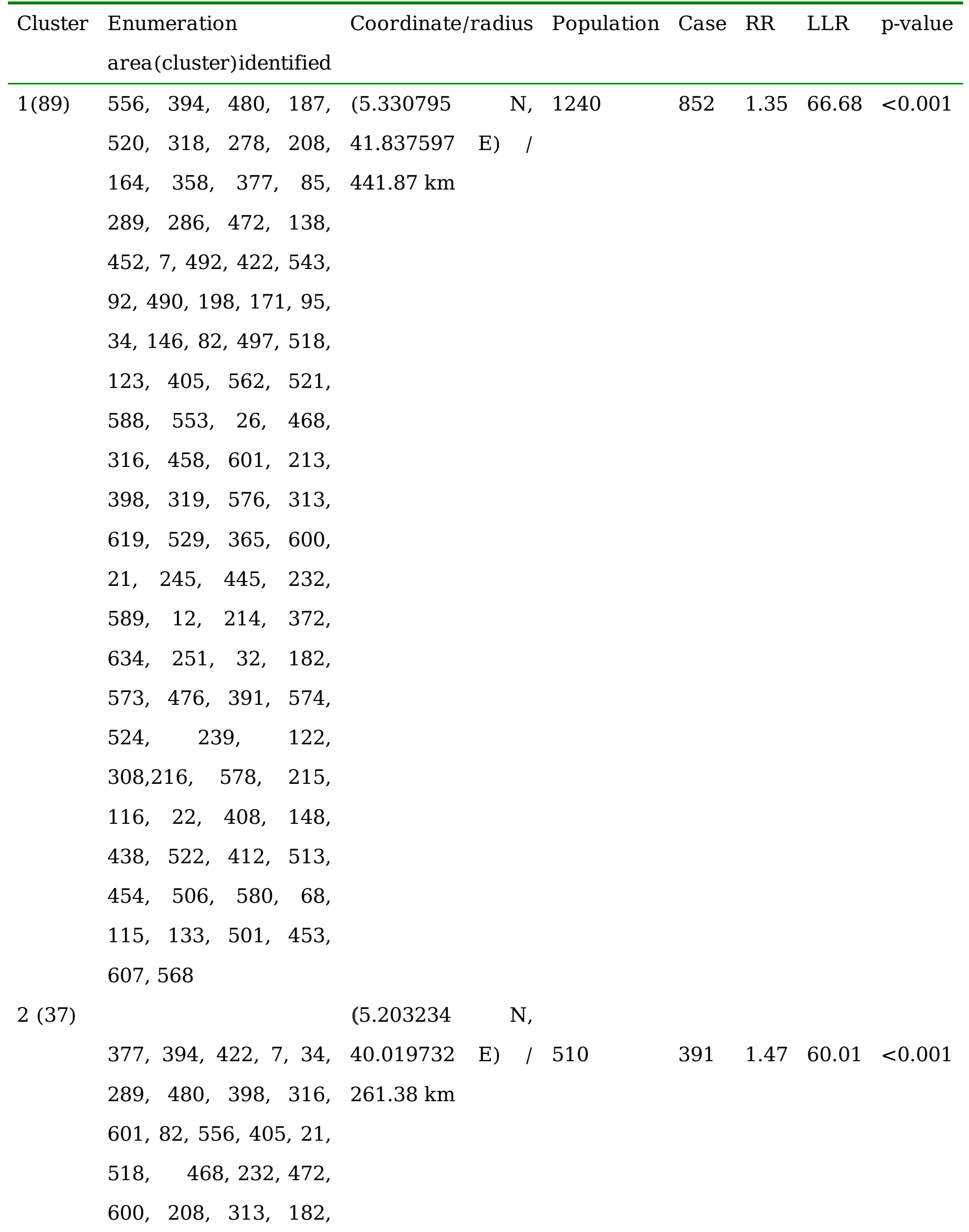


$445,574,32,576$,

286, 634, 26,365, 452,

$520,12,215,216,408$

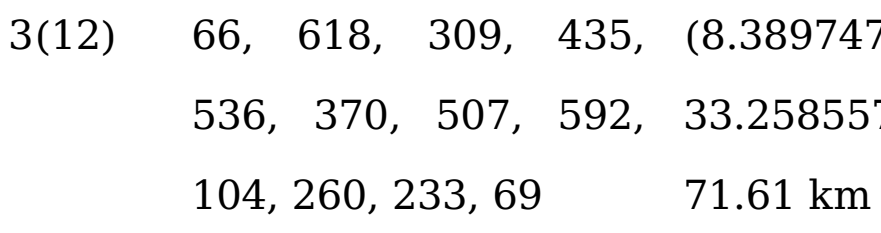

4(49) 630, 378, 269, 629, 7.717178

N, 587

132

$1.7 \quad 46.72<0.001$

77, 146, 92, 490, 543, 46.991580

E) $/$

$171,492, \quad 198, \quad 95,555.85 \mathrm{~km}$

497, 458, 588, 553,

$521,138,214,33$,

573, 251, 239, 116,

85, 358, 22,164, 527,

568, 277, 439, 64, 57,

278, 210, 8, 186, 566,

1, 318, 622,436, 212,

454,501

5(10) 1, 566, 622, 186, 307,

$(9.505470$

N, 142

$117 \quad 1.54 \quad 25.76<0.001$

436, 212, 8, 210, 419 42.438628

E) $/$

$33.79 \mathrm{~km}$

$6(42)$

477, 325, 207, 437, (7.173968

N, 509

343

$1.27 \quad 20.07<0.001$

$376,154,168,177,35.802680$

E) 1

$552,459,371,243,170.61 \mathrm{~km}$

465, 299, 526, 554,

197, 46, 586, 489,

119, 338, 76, 326,

555, 470, 337,

$432,486,447,448,62$,

$306,227,446,411$, 
219, 558, 270, 593,

265,406

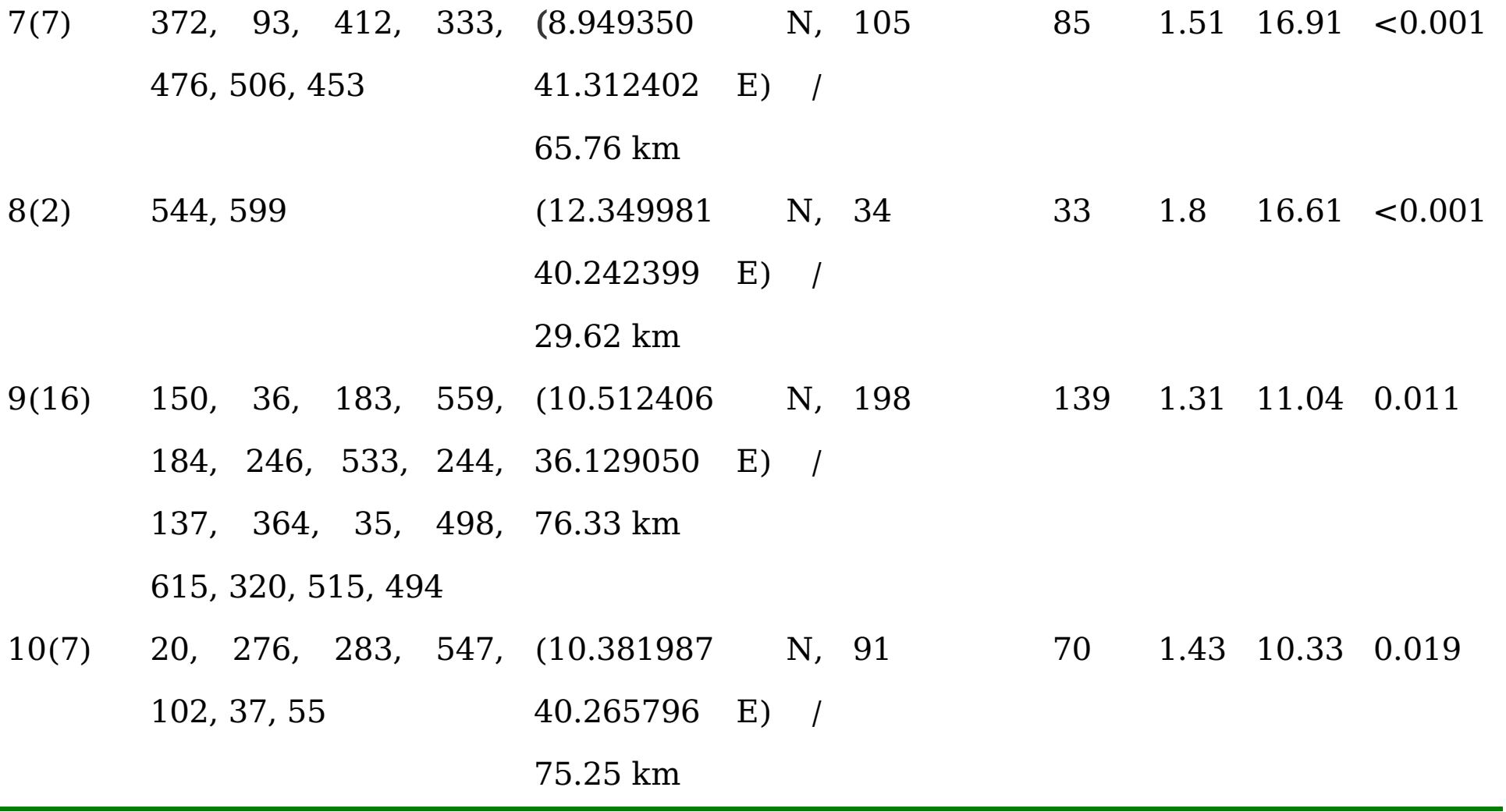

Table 3: Multivariable multilevel logistic regression analysis result of both individual and community level factors associated with iron supplementation among pregnant women in Ethiopia, EDHS 2016. 


\begin{tabular}{|c|c|c|c|c|c|c|}
\hline \multirow{2}{*}{$\begin{array}{l}\text { Variables } \\
\text { Age }\end{array}$} & \multirow[t]{2}{*}{ Null model } & \multicolumn{2}{|c|}{$\begin{array}{l}\text { Model II } \\
\text { AOR(95\%CI) }\end{array}$} & $\begin{array}{l}\text { Model III } \\
\text { AOR(95\%CI) }\end{array}$ & \multicolumn{2}{|c|}{$\begin{array}{l}\text { Model IV } \\
\text { AOR(95\%CI) }\end{array}$} \\
\hline & & & & & & \\
\hline \multirow[t]{2}{*}{$15-19$} & & 1.27 & {$[0.78-$} & & 1.38 & [0.85- \\
\hline & & $2.06]$ & & & 2.23] & \\
\hline \multirow[t]{2}{*}{$20-24$} & & 1.34 & [0.87- & & 1.41 & [0.92- \\
\hline & & 2.06] & & & $2.16]$ & \\
\hline \multirow[t]{2}{*}{$25-29$} & & 1.21 & {$[0.80-$} & & 1.29 & {$[0.85-$} \\
\hline & & 1.83] & & & 1.95] & \\
\hline \multirow[t]{2}{*}{$30-34$} & & 1.01 & {$[0.67-$} & & 1.06 & {$[0.71-$} \\
\hline & & 1.52] & & & 1.59] & \\
\hline \multirow[t]{2}{*}{ 35-39 } & & 0.88 & [0.59- & & 0.89 & [0.59- \\
\hline & & 1.33] & & & 1.33] & \\
\hline \multirow[t]{2}{*}{$40-44$} & & 0.71 & [0.46- & & 0.70 & {$[0.45-$} \\
\hline & & 1.09] & & & $1.07]$ & \\
\hline $45-49$ & & 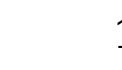 & & & 1 & \\
\hline \multicolumn{7}{|l|}{ Residence } \\
\hline Rural & & & & 1 & & \\
\hline \multirow[t]{2}{*}{ Urban } & & & & {$[1.42-$} & 1.24 & {$[0.92-$} \\
\hline & & & & 2.33] & 1.66] & \\
\hline
\end{tabular}

Region

Somali

Tigray

Afar

Amhara

Oromia

Benishangul-Gumuz

\begin{tabular}{lrll}
\multicolumn{1}{r}{1} & \multicolumn{2}{c}{1} \\
9.18 & {$[6.35-$} & 5.35 & {$[3.73-$} \\
$13.28]$ & & $7.69]^{* *}$ & \\
1.75 & {$[1.22-$} & 1.61 & {$[1.13-$} \\
$2.51]$ & & $2.28]^{* *}$ & \\
3.08 & {$[2.19-$} & 2.07 & {$[1.47-$} \\
$4.33]$ & & $2.91]^{* *}$ & \\
1.25 & {$[0.89-$} & 0.81 & {$[0.58-$} \\
$1.75]$ & & $1.14]$ & \\
2.37 & {$[1.62-$} & 1.37 & {$[0.94-$}
\end{tabular}




\section{SNNPR}

Gambelia

Harari

Addis Ababa

Dire dawa

Wealth index

Poorest

Poorer

Middle

Richer

Richest

Community

education

Lower community education

Higher community

education

Occupational status

Not working

Working

women's 
A big problem

Not a big problem

Parity

0-4

5-9

$10+$

Family size

1-4

5-9

$10+$

ANC visit

0-3 times

4+times

Media exposure

Not have exposure

Have exposure

Constant

Log likelihood (LL)

Deviance

$5-9$

1.43

1.61]

1

[1.26- 1.32

[1.16-

1.50]**

1

1.04 [0.88-

1.07

[0.91-

1.23]

1.26]

1.05 [0.70-

1.10

[0.74-

1.56]

1.65]

1

0.98 [0.85-

1.01 [0.87-

1.13]

1.16]

0.97 [0.73-

1.06

[0.80-

1.29]

1.41]

\begin{tabular}{clc}
1 & \multicolumn{2}{c}{1} \\
$3.9[3.43-4.44]$ & 3.66 & {$[3.21-$} \\
& $417]^{* *}$
\end{tabular}


PCV

Ref

0.41

0.59

0.67

MOR

$2.9(2.65,3.2)$

$2.27(2.09,2.49)$

$1.97(1.84,2.5) \quad 1.84(1.70,2.00)$

Key: AOR: Adjusted odds ratio; CI: confidence interval; ICC: intra-cluster correlation; MOR: median odds ratio; 1 : reference group; p-value 0.05-0.01*: p-value $<0.01 * *$ : ANC; antenatal care visit

\section{Figures}

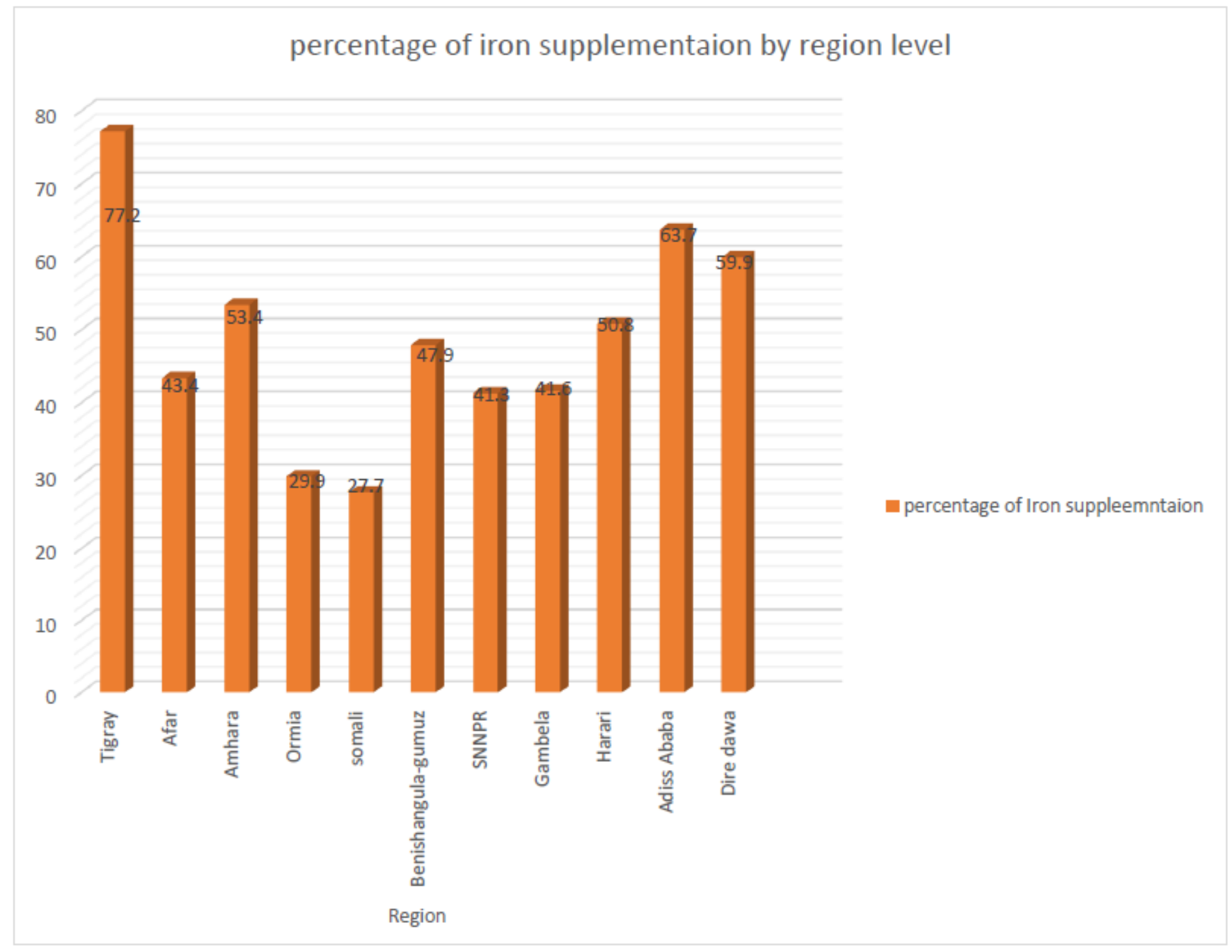

Figure 1

Regional prevalence of iron supplementation among pregnant women in Ethiopia, 2016 
Spatial distribution of Iron supplementation among pregnant women in Ethiopia 2016

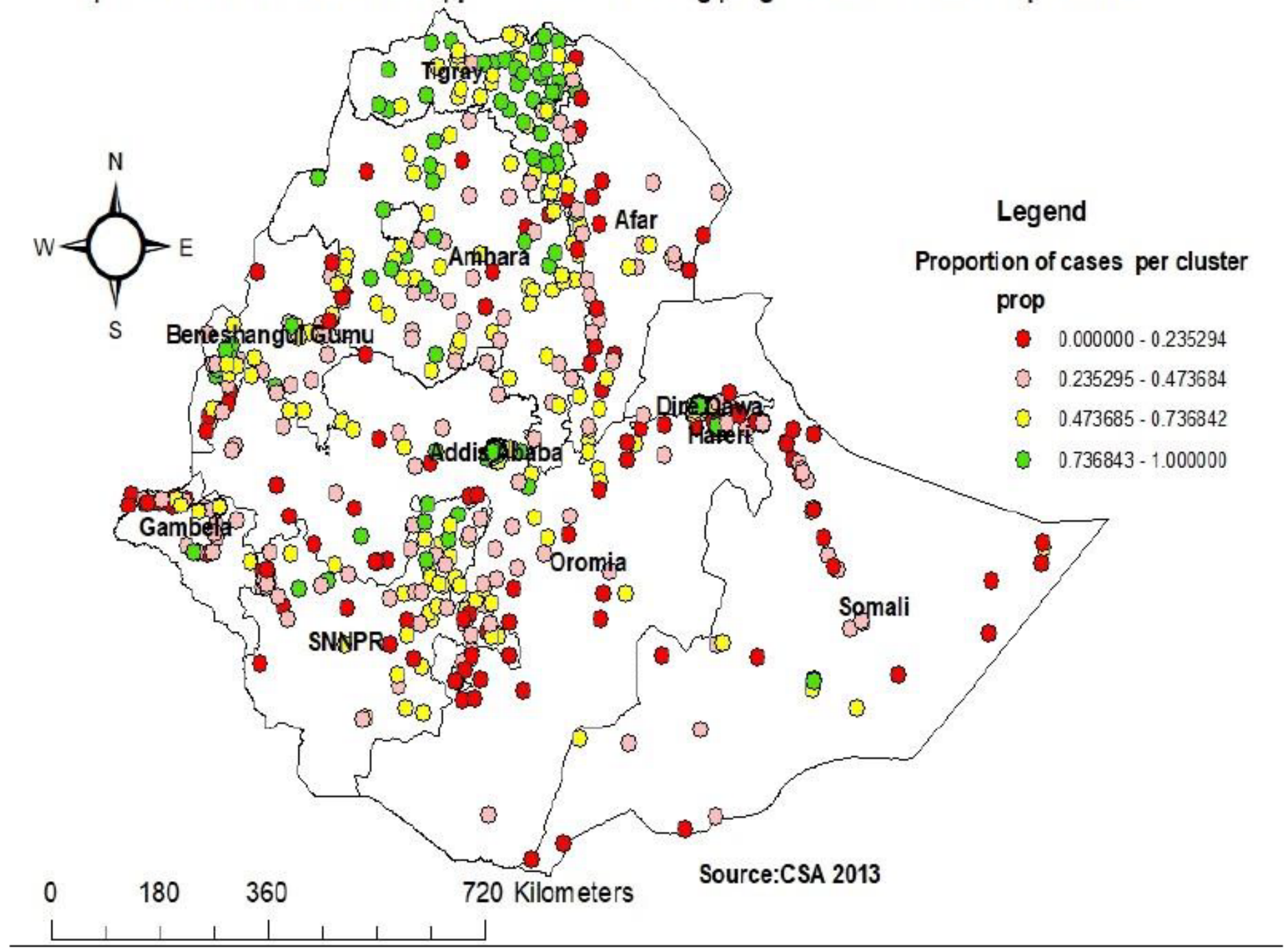

Figure 2

Spatial distribution of Iron supplementation among pregnant women in Ethiopia, 2016 
Hot spot analysis poor iron supplementation among pregnant women in Ethiopia,2016

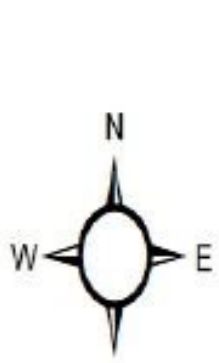

S
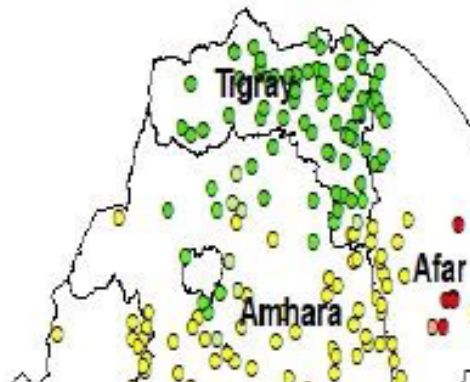

800
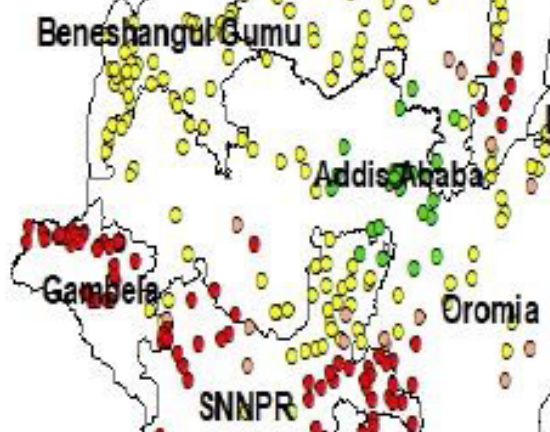

SNNPR
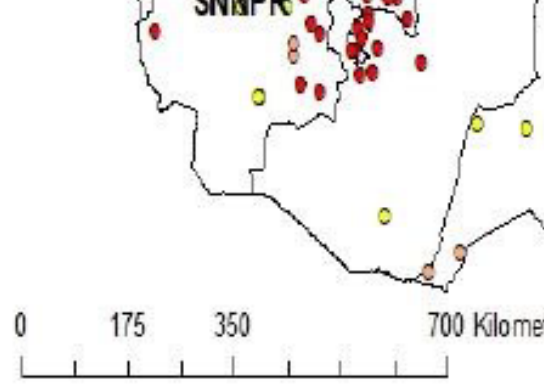

\section{Legend}

Hot Spot analysis

Gi_Bin

- Cold Spot - $99 \%$ Confidence

- Cold Spot-95\% Confidence

- Cold Spot-90\% Confidence

- Not Significant

- Hot Spot-90\% Confidence

- Hot Spot-95\% Confidence

- Hot Spot-99\% Confidence

Source:CSA 2013

\section{Figure 3}

Hot spot and cold spot identifications of Iron supplementation among pregnant women in Ethiopia, 2016. 
Interpolation of iron supplementation among pregnant women in Ethiopia,2016
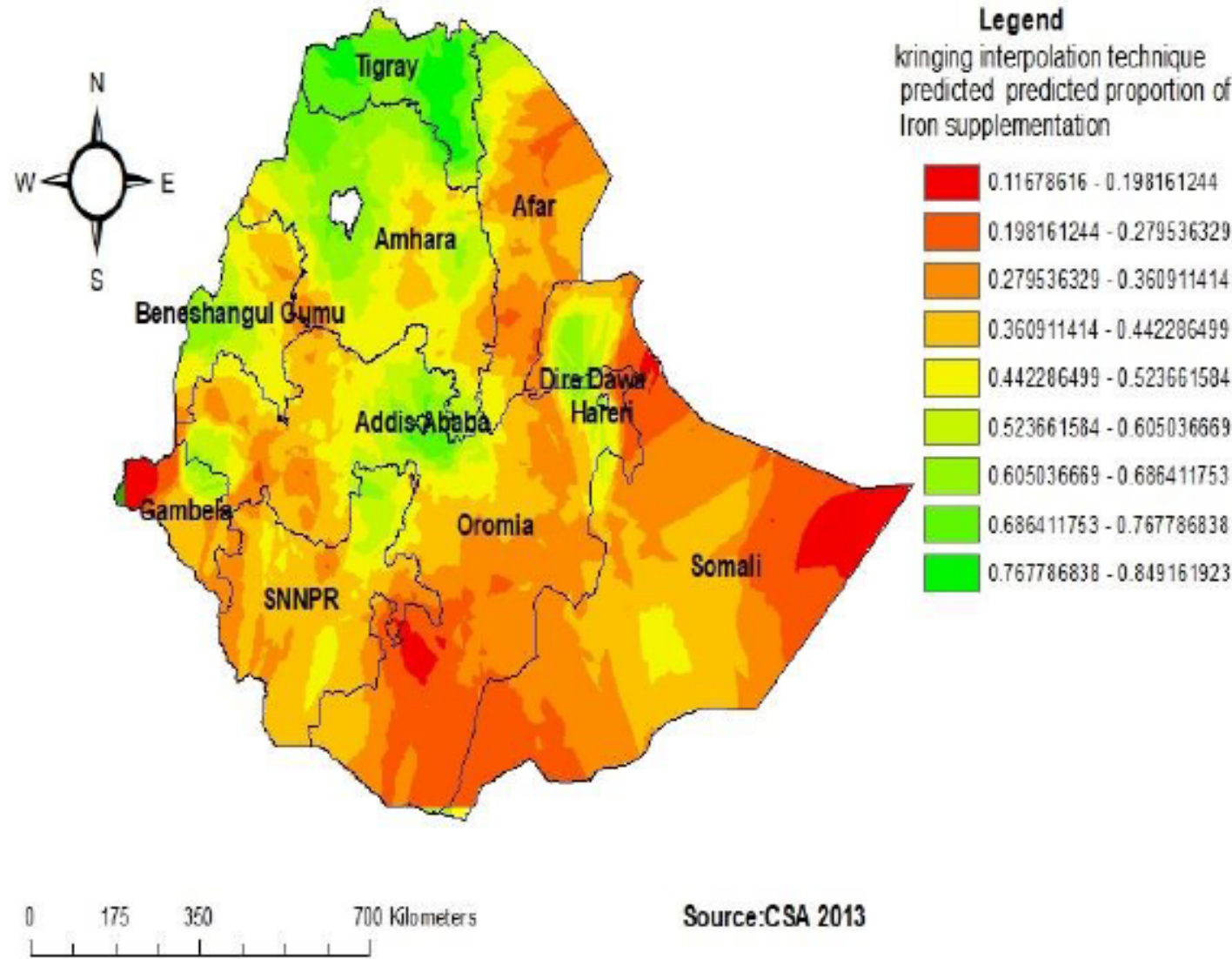

Source:CSA 2013

*red color indicates: predicted high risk areas; Green color: low risk areas

\section{Figure 4}

Kriging interpolation of iron supplementation among pregnant women in Ethiopia, 2016. 


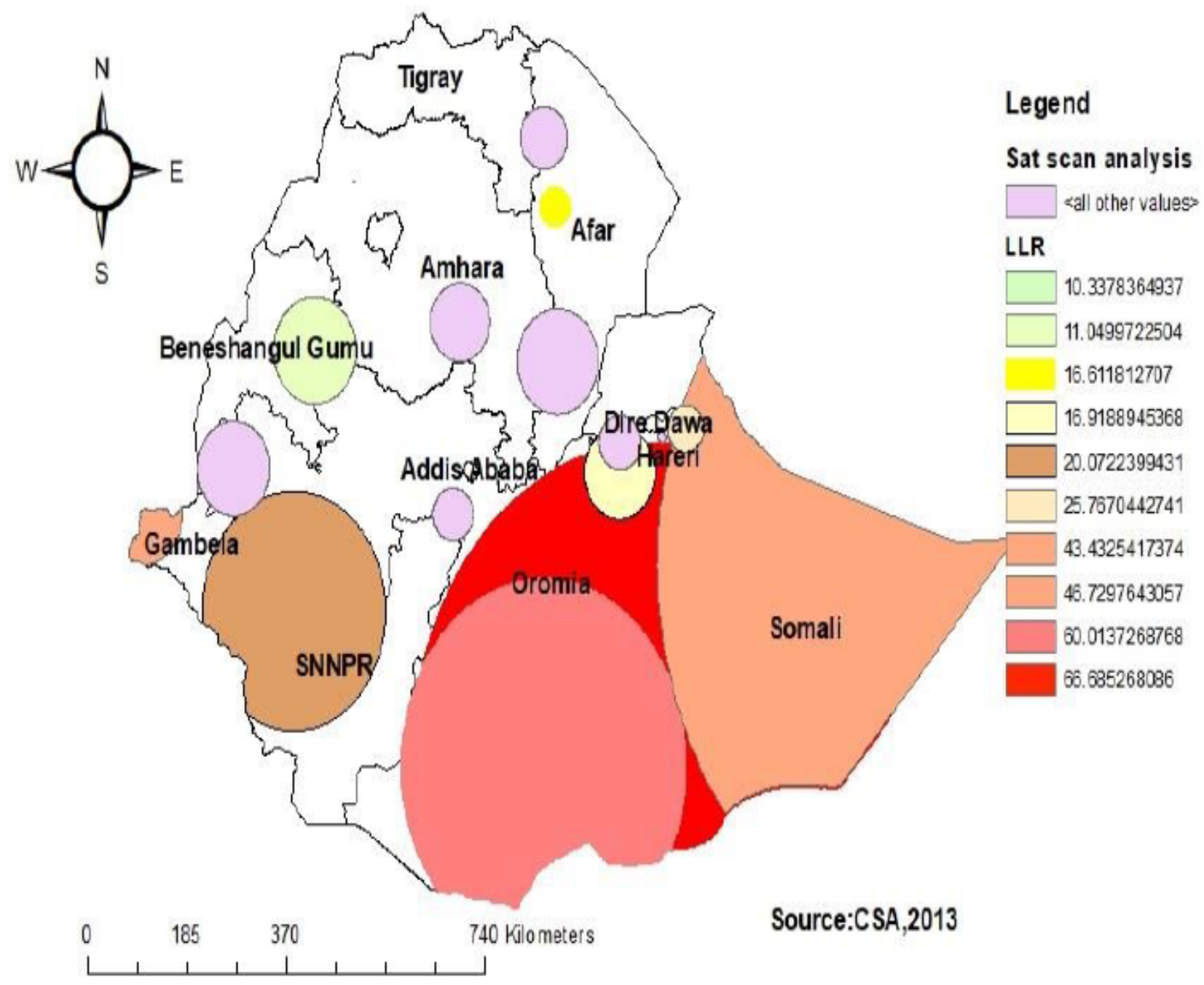

Figure 5

Spatial Sat Scan analysis of iron supplementation among pregnant women in Ethiopia, 2016 